\title{
Familia y Redes Sociales: un binomio controvertido
}

\author{
Isabel Dans Álvarez de Sotomayor (*), Pablo César Muñoz Carril (**) y Mercedes González Sanmamed (**) \\ ${ }^{*}$ ) Universidad de Vigo, $\left.{ }^{* *}\right)$ Universidad de Santiago de Compostela, $\left({ }^{* * *}\right)$ Universidad de A Coruña
}

\begin{abstract}
RESUMEN
Las redes sociales poseen una gran importancia en la vida de los adolescentes y la labor de las familias resulta imprescindible para aprovechar las posibilidades que ofrecen y frenar los peligros que suscitan. En este estudio se analizan, a través del método de encuesta, las opiniones de 1144 alumnos de cuarto de educación secundaria sobre la mediación parental en el acceso y uso de las redes sociales. Tras análisis descriptivos y no paramétricos, los resultados revelan que los menores se inician en el uso de redes sociales a través de su grupo de amigos, constatándose la escasez de medidas de control familiar frente al uso de este tipo de herramientas. Asimismo, el acceso y tiempo de conexión a las redes sociales guardan relación con el rendimiento escolar y el género. También se identifican diferencias importantes en cuanto a la percepción que familias, docentes y alumnado tienen sobre las redes sociales, siendo estos últimos los que manifiestan una visión más positiva. Entre las conclusiones cabe señalar la necesidad de que las familias desarrollen medidas educativas adecuadas que propicien que los menores realicen un uso responsable y seguro de las redes sociales.
\end{abstract}

Palabras Clave: Redes sociales, familia, educación secundaria, adolescencia, educación digital.

\section{Family and social networks: controversial binomial}

\section{ABSTRACT}

Social networks have great importance in the lives of adolescents and the involvement of their families is essential to take full advantage of the possibilities offered and stop the dangers that may arise. This study analyses, through a survey method, the opinions of 1144 secondary school students on parental mediation of access and use of social networks. After descriptive and non-parametric analysis, the results reveal that the minors start using social networks influenced by their group of friends, confirming the lack of family control measures of the use of this type of tool. Likewise, access and time of connection to social networks are linked to school performance and gender. Important differences have also been identified in terms of the perception that families, teachers and students have of social networks, the latter having a more positive view than that of their parents and teachers. In conclusion, it is necessary for families to know what use their children make of social networks, and to develop appropriate educational measures that promote responsible and safe uses.

Keywords: Social networks, family, secondary education, adolescence, digital education.

\section{Introducción}

\subsection{La labor educativa de la familia en la sociedad en red}

La sociedad doméstica es la comunidad educativa más antigua, cuyos miembros juegan roles decisivos que determinan el carácter y la orientación de la educación familiar. El desarrollo de las sociedades postindustriales ha generado cambios decisivos que han afectado a la estructura y funciones de la familia, pero a día de hoy sigue siendo el primer agente de socialización, cuya influencia resulta fundamental a lo largo de la vida. Esta tarea está ampliamente reconocida en diversos tratados y normativas como, por ejemplo, la Declaración Universal de los Derechos
Humanos, la Convención Internacional de los Derechos del Niño o la propia Constitución Española.

En la actualidad, cualquier referencia a la familia debe plantearse desde el respeto a la diversidad y el reconocimiento del carácter único de cada sistema familiar. En cualquier caso, resulta plenamente aceptada la orientación natural de las familias hacia el cuidado de los hijos y su responsabilidad en ayudarles en su desarrollo personal y equilibrio emocional, independientemente de los sucesos, fenómenos y circunstancias por las que atraviesan y las vicisitudes a las que tengan que enfrentarse (Ródenas-Rigla, Fombuena y Pérez-Cosín, 2017).

Sin menosprecio de la importancia de la familia, queda fuera de duda la influencia de otros agentes con los que los menores 
entran en contacto y las repercusiones que suscitan en la configuración de su personalidad. Así, además del entorno escolar, cabe reconocer que las actividades lúdicas y las experiencias audiovisuales afectan de manera decisiva en las diversas etapas de crecimiento de las personas (Bronfenbrenner, 1994; Moratalla, 2008). Los medios de comunicación, internet y, particularmente, las redes sociales, tienen un papel primordial en la configuración de la identidad de los adolescentes. Teniendo en cuenta que la cifra de smartphones ha ascendido de un $41 \%$ en 2012 a un $89 \%$ en 2018 (Common Sense Media, 2018), casi podría afirmarse que vivimos en una sociedad donde ya hay más móviles que personas. Pero, además, la conectividad está disponible a todas horas y desde cualquier lugar, y ello está generando cambios importantes en las formas de comportarse, de pensar y de ser (Ballesta, Lozano, Cerezo y Soriano, 2015).

Por todo ello, en el contexto tecnológico actual, los jóvenes requieren ayuda para actuar de manera responsable, fluida y diversificada en su entorno digital y aprender a protegerse en cada momento social de su vida cotidiana en la red Chan, Walker y Gleaves (2015) indican que los padres y otros adultos influyen en los patrones de uso y las motivaciones, además de generar retroalimentación, acerca de todo lo que sale al encuentro de los adolescentes en internet. Se trata de una influencia limitada, pero no se puede despreciar su aportación y la posibilidad de actuar como un modelo potencialmente importante para los jóvenes.

Desde esta perspectiva, resulta imprescindible abordar estudios relacionados con el rol de las familias respecto a las redes sociales de sus hijos.

\subsection{Adolescencia en el universo digital}

La era de la distracción, como se ha dado en denominar nuestra época, ha sido estudiada por el Pew Research Center (2018). Esta institución ha revelado que el 54\% de los adolescentes de EE.UU. reconocen que pasan demasiado tiempo con sus teléfonos móviles, y dos tercios de los padres expresan preocupación por las horas que sus hijos están frente a las pantallas. En este sentido, diversos autores (Carr, 2011, Christakis et al., 2010; Kuppuswamy y Narayan, 2010; Naval, Serrano-Puche, Sádaba y Arbués, 2016) se refieren a la necesidad de la desconexión y, como evidencian los experimentos al respecto (Moeller, Powers y Roberts, 2012), esta medida puede suscitar un importante revulsivo ante la dependencia exagerada de las pantallas.

Los primeros retratos de familia, de la actual generación de jóvenes, son subidos a las redes sociales por sus propios padres. A través de este fenómeno, denominado "sharenting" (Livingstone, Blum-Ross y Zhang, 2018), se difunden datos, imágenes y vídeos que van configurando el territorio donde se forja el estilo de vida de los adolescentes actuales. A través de esta información compartida se construye su desarrollo psicosocial como autorrepresentación identitaria y medio de exploración del contexto (Valkenburg y Peter, 2011). Sirve indirectamente para conocerse, presentarse y, en suma, construir su propia identidad. Pero, además, las relaciones están marcadas por la pertenencia a los grupos digitales, donde la marca personal puede fabricar un "influencer" o convertirles en seguidores o fans de personas que inspiran conductas, consumos e ideas.

Cabe señalar que los adolescentes han ganado un gran protagonismo social y el reconocimiento de las competencias que adquieren en el uso de la tecnología abruman a las familias y a sus educadores, generando una brecha digital intergeneracional (Salcines-Talledo, Ramírez y González-Fernández, 2018). Pero, la competencia técnica ni está tan desarrollada ni es la única que preocupa a los adultos, quienes pueden llegar a mitificar a los jóvenes por sus habilidades tecnológicas (Busquet, Medina y Ballano, 2013; Garrido, Busquet y Munté, 2016).

\subsection{Adolescentes, familias y redes sociales}

Desde el éxito de MySpace en 2003, han aparecido y desaparecido redes sociales de uso masivo, como es el caso de Tuenti en España. Hoy en día, entre las más populares, destacan Instagram y Snapchat. A su lado tienen un papel principal en la comunicación social las herramientas de mensajería instantánea, como Whatsapp, con funcionalidades que asemejan a las propias redes sociales. La euforia inicial ha dado paso a un consumo más limitado, con una gran presencia de fines publicitarios en torno a productos, marcas y opinión pública en general. La regulación de la edad mínima para su uso ha sido modificada y varía entre los 14 y los 13 años. La convergencia de medios multiplica su presencia en la televisión, las series o la prensa digital. Los contenidos expresados en texto, imágenes o vídeo forman parte de la vida cotidiana y son objeto de controversia legal. El derecho a la intimidad o a la libertad de expresión son algunas de las banderas ondeadas para defender a los usuarios de las redes sociales. La privacidad constituye una de las grandes preocupaciones de la sociedad, dado que los datos circulan por los dispositivos muchas veces de forma involuntaria (Kirschner y Karpinski, 2010; Thunseethan y Kuhanesan, 2014).

Las redes sociales resultan cada vez más accesibles a menores y la legislación camina despacio para hacer frente a los riesgos que pueden derivarse de algunas prácticas inapropiadas y/o abusivas. Así pues, junto a las enormes posibilidades que ofrecen en estos entornos digitales, cabe tomar conciencia también de ciertos peligros como la hipersexualización (Vázquez, Hurtado y Baños, 2018), las adicciones (Christakis, Fowler, Diéguez, Vidal y Schmid, 2010; Durkee et al., 2012; Salmerón, 2015; Rial, Gómez, Braña y Varela, 2014) o el ciberbullying (INTECO, 2011), entre otros.

En plena era de la información y del conocimiento, se constatan diferencias sustanciales entre la actitud de los jóvenes y sus familias hacia el mundo digital. Los adultos se sienten confundidos (Garrido et al., 2016; Ruiz-Corbella y De-Juanas, 2013), aunque los estudios indican que cuando aumenta la frecuencia de uso y se incrementa el dominio de las herramientas, se produce una mejora en la percepción de los usuarios (Chan, et al. 2015).

Los datos indican que las familias controlan las redes de sus hijos de distinta manera. El informe europeo de Livingstone y Haddon (2011) muestra que el 77\% de los padres no realiza seguimiento de las páginas visitadas por los hijos. También Colás, González y De Pablos (2013, p. 19) indican que "solo el 22,3\% tiene unas normas de uso; el resto puede acceder a la Red sin ningún tipo de limitación". En Galicia, las familias se muestran divididas: en el 46\% de los jóvenes encuestados existe control parental y la mayoría (un 78\%) nunca o casi nunca discute con sus padres en la relación al uso de las redes sociales (Rial et al., 2014).

Las preocupaciones de los padres son materia principal de los estudios sobre redes sociales y familia (Shin y Kang, 2016). Temas como la violencia -en forma de acoso en línea-, junto al tiempo de uso, la descarga de programas o aplicaciones maliciosas, el engaño y el contacto con desconocidos o la exposición excesiva de información personal, son las cuestiones que inquietan en gran medida a las familias. En Estados Unidos un estudio muestra que 3 de cada 4 padres afirman que las redes sociales no protegen la privacidad de sus hijos (Common Sense Media, 2010). Los datos que ofrecen las empresas de seguridad en línea resultan sorprendentes. El informe de McAffe (Davis, 2018) afirma que el $30 \%$ de los padres sube fotos de sus hijos una vez 
al día, con lo que se fomenta la sobreexposición de los menores en las redes. Norton (2018) realza el interés de los padres por la seguridad en la red fruto de sus propias experiencias. Así, el $87 \%$ valora osado dejar ordenadores y dispositivos móviles a sus hijos sin ninguna supervisión. A pesar de este dato, el 57\% les permite hacerlo sin vigilancia alguna.

Mullen (2016) mide la respuesta de los adolescentes a la presencia de sus padres en cuanto a tres variables: influencia en su privacidad, calidad relacional y presión del grupo. Los resultados que arroja su estudio muestran que los adolescentes son indiferentes al control parental e incluso que puede resultar contraproducente. La mediación centrada en el tiempo de uso ha sido destacada por diversos autores (Bringué y Sádaba, 2011; Shin y Kang, 2016). Se mantiene la paradoja entre la preocupación y el patrón de conducta: el miedo a los peligros de la sobreexposición no se traduce en una protección adecuada sobre los contenidos, sino sobre el tiempo de conexión (Taddicken, 2014).

También resulta preocupante la falta de habilidades personales para la relación presencial, como exposición no controlada al otro. La percepción que tienen los jóvenes acerca del modo de comunicarse manifiesta su reticencia hacia la interrelación cara a cara. El extremo de esta conducta pasa del aislamiento a la intimidad, por lo que la percepción de felicidad que ofrecen las redes sociales es variable (López Moratalla, Bernar, Sueiro y Valderas, 2011). Los adolescentes son más propensos a afirmar que estas les hacen sentirse mejor: menos solo, deprimido o ansioso y con más confianza, autoestima y popularidad. "Likeability" es una de las gratificaciones que se obtienen del uso de las redes sociales. García-Ruíz, Tirado y Hernando (2018) concluyen que los estudiantes de Secundaria usan las redes sociales con el propósito de propiciar una mayor presencia social y, sobre todo, les preocupa sobremanera su propia imagen. Por otra parte, cabe señalar que una utilización inconsciente de las redes aleja a los adolescentes de tomar partido en la configuración de su propio mundo.

Otra incongruencia se produce en cuanto a la utilización del móvil para el aprendizaje. La regulación sobre este dispositivo lleva a su prohibición en el entorno escolar, salvo un uso pedagógico puntual. Sin embargo, las familias se oponen por el miedo a la libertad de acceso a contenidos inadecuados, a pesar de que los estudiantes están altamente equipados por sus progenitores (Chan et al., 2015). Se les dota de medios y conectividad en sus hogares y en los centros educativos se bloquean estas posibilidades. Pero frente a estos y otros problemas, dificultades y desafíos, la inclusión de redes sociales en el ámbito educativo constituye un fenómeno en aumento (González Sanmamed, Muñoz y Dans, 2017) y se recomienda su incorporación en procesos de enseñanza y práctica educativa (García-Ruíz, Tirado y Hernando, 2018; Hamid, Waycott, Kurnia y Chang, 2015) por los beneficios formativos que son capaces de aportar a los estudiantes como recurso educativo (Bicen y Uzunboylu, 2013; Hamid, Waycott, Kurnia y Chang, 2015; Junco, 2015; Jung Lee, 2009; Vázquez-Martínez y Cabero, 2014). Por su parte, Muñoz-Carril, Dans-Álvarez-de-Sotomayor y González-Sanmamed (2019) han estructurado la literatura científica sobre redes sociales y educación en tres niveles: usos de las redes sociales para la enseñanza y el aprendizaje, usos institucionales y políticas educativas sobre alfabetización digital. Desde esta triple perspectiva se van desvelando las oportunidades y las exigencias que suscita el uso de las redes sociales tanto a nivel de aula como de centro educativo, y sus implicaciones en el ámbito de la política educativa y en la sociedad en general.

Para dar respuesta a los desafíos y controversias que suscitan las redes sociales, las investigaciones coinciden en la necesidad de incrementar la formación digital (Almu y Buhari, 2014; Bus- quet et al., 2013; Kirschner y Karpinski, 2010; Salcines-Talledo, Ramírez y González-Fernández, 2018; Thuseethan y Kuhanesan, 2014). A pesar de las dificultades que entraña un contexto tan cambiante, ubicuo y difuso como el actual, se han elaborado diversos programas de asesoramiento y orientación, tanto por parte de las administraciones públicas como de organizaciones privadas. En esa línea, las principales redes sociales han publicado guías para padres y educadores (Tuenti en 2013, Facebook en 2016 e Instagram en 2018) con ayudas técnicas para administrar la privacidad, las interacciones o el tiempo de uso, además de consejos para la conversación familiar en torno a este fenómeno.

Los adolescentes, sus familias y las redes sociales constituyen los vértices de un triángulo en el que se vierten muchas de las actuales contradicciones de la sociedad digital, se ponen de manifiesto los retos a los que hay que dar respuesta y se perfilan algunas de las posibles respuestas que podrían explorarse. Bajo esta perspectiva, se ha desarrollado un estudio a través del que se ha recogido la percepción de un grupo de adolescentes acerca de la valoración que sus familias realizaban de las redes sociales.

\section{Metodología}

\subsection{Población y muestra}

Sobre una población de 1.792 estudiantes de $4^{\text {o }}$ curso de enseñanza secundaria de la ciudad de A Coruña, distribuidos en 31 centros educativos, se recogieron mediante un muestreo por conveniencia 1.144 encuestas de 29 instituciones.

\subsection{Instrumento}

Se utilizó un diseño ex post facto basado en el método de encuesta (McMillan y Schumacher, 2005), empleando un cuestionario ad hoc compuesto por un total de 251 ítems estructurados en cinco bloques temáticos: datos de identificación de los participantes; usos de internet y otras tecnologías; utilización de redes sociales; posibilidades de uso de las redes sociales para el aprendizaje escolar y peligros y ventajas de las redes sociales. Las respuestas que se analizan en este trabajo corresponden a los bloques de datos iniciales de identificación de la muestra, usos de Internet y otras tecnologías, utilización de redes sociales y opiniones, percepciones y valoraciones.

Con el fin de asegurar las condiciones psicométricas adecuadas, en la elaboración del cuestionario se atendieron cuestiones como la validez de contenido, de constructo y consistencia interna. El instrumento fue validado por un panel de 5 expertos en metodología de investigación y aplicación de la tecnología a la educación, abordando aspectos como la univocidad, pertinencia e importancia de cada ítem. También se aplicó a un grupo piloto. Para medir la fiabilidad se utilizó alpha de Cronbach que arrojó un resultado elevado de 0,937 .

Antes de la aplicación del cuestionario, los padres y los estudiantes fueron informados del objetivo del estudio y dieron su consentimiento para participar. La recogida tuvo lugar en su propia aula de forma voluntaria en presencia del profesorado. El cuestionario se distribuyó en papel entre los estudiantes, estando presentes alguno de los investigadores para solucionar cualquier duda que pudiera surgir. Tras su aplicación, los datos obtenidos fueron analizados con el programa IBM SPSS.

\subsection{Procedimiento y análisis de datos.}

Los análisis desarrollados se articulan a través de estadística descriptiva y no paramétrica. Mediante los análisis descriptivos 
se ha pretendido recoger, describir, organizar y sintetizar los datos observados (Vilà y Bisquerra, 2004). Dado el carácter nominal de las variables analizadas se ha prestado especial atención a aspectos clave como las frecuencias y porcentajes mediante su representación en diagrama de sectores.

Respecto a los análisis no paramétricos, el propósito ha sido identificar diferencias significativas entre las variables objeto de estudio. Para ello se han utilizado análisis bivariados a través del contraste Chi-cuadrado.

Por otra parte, los diferentes análisis planteados pivotan en torno a los siguientes objetivos de investigación:

- Identificar de qué forma los adolescentes se han iniciado en el ámbito de las redes sociales y, más específicamente, quienes los han invitado por primera vez a formar parte de alguna de ellas.

- Conocer la percepción de los menores en torno a quien les limita o decide (y en qué medida) el tiempo de conexión a las redes sociales.

- Identificar si las redes sociales son utilizadas por los padres/madres como un elemento de premio o castigo según el comportamiento de sus hijos.

- Conocer desde el punto de vista de los adolescentes si sus padres/madres les permitirían conectarse a las redes sociales en función de los usos que hacen de las mismas.

- Analizar la imagen que los estudiantes de $4^{\circ}$ de Educación Secundaria tienen de las redes sociales y cuál es la percepción que ellos consideran que tienen sus padres y profesores hacia estas herramientas

\section{Resultados}

\subsection{Análisis descriptivos de las variables objeto de estudio}

Seguidamente se muestran los resultados derivados del análisis de las 5 variables listadas a continuación:

- ¿Quién te invitó a formar parte de tu primera Red Social?

- ¿Quién decide el tiempo que puedes conectarte a las redes sociales?

- ¿Tus padres deciden si te dejan conectarte a las Redes Sociales como premio o castigo por tu comportamiento, tus calificaciones, etc.?

- Si tus padres supieran qué haces en las Redes Sociales ¿te dejarían conectarte a ellas?

- ¿Cuál es la imagen que los adolescentes tienen de las redes sociales y qué percepción tienen acerca de la opinión que tienen sus padres y profesores sobre estas herramientas?

Tal y como se refleja en la figura 1, el alumnado encuestado ha señalado que son preferentemente sus amigos (72,5\%), seguido de los familiares (12,2\%) y de los compañeros de clase $(8,4 \%)$ quienes en primera instancia los han invitado a formar parte de su primera red social.

Por otra parte, ante la pregunta relativa a "¿quién decide el tiempo que puedes conectarte a las redes sociales?" (ver figura 2 ), un elevado porcentaje de estudiantes ha respondido que lo deciden ellos mismos (55,2\%). También resulta revelador el hecho de que un $15,9 \%$ de los encuestados hayan manifestado que sus padres no limitan su tiempo de conexión.

Sorprende, asimismo, que un $68,9 \%$ de los encuestados afirman que sus padres nunca o casi nunca utilizan las redes sociales como mecanismo que sirva de premio o castigo en relación con su comportamiento o rendimiento académico (figura 3). Por con-

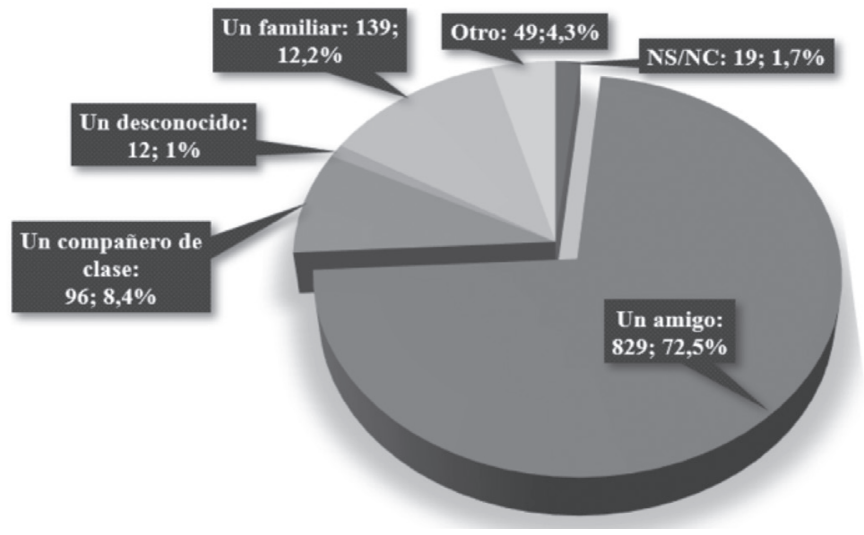

Figura 1. Frecuencias y porcentajes relativos al item ¿quién te invitó a formar parte de tu primera Red Social?

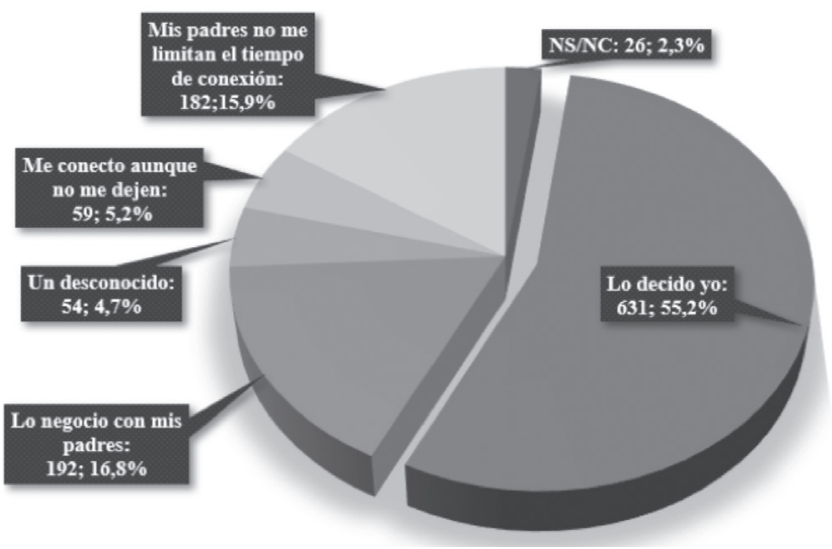

Figura 2. Frecuencias y porcentajes del item "¿quién decide el tiempo que puedes conectarte a las redes sociales?

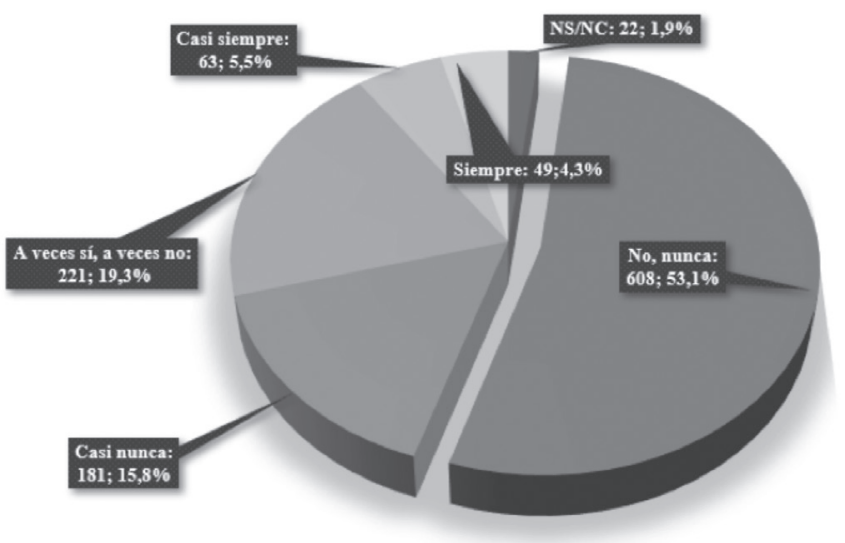

Figura 3. Frecuencias y porcentajes del ítem " ¿Tus padres deciden si te dejan conectarte a las Redes Sociales como premio o castigo por tu comportamiento, tus calificaciones, etc.

siguiente, no parece existir un estricto filtro parental referente al uso de este tipo de herramientas por parte de los adolescentes.

Otra cuestión planteada en la investigación se centró en conocer si los padres dejarían a sus hijos conectarse a las redes sociales si supiesen realmente lo que hacen en ellas. En este sentido, un $54,6 \%$ de los adolescentes respondió que sus progenitores siempre les dejarían conectarse, frente a solo un 5,1\% que han señalado que nunca se lo permitirían. En la figura 4 se pueden observar con mayor detalle las frecuencias y porcentajes obtenidos. 


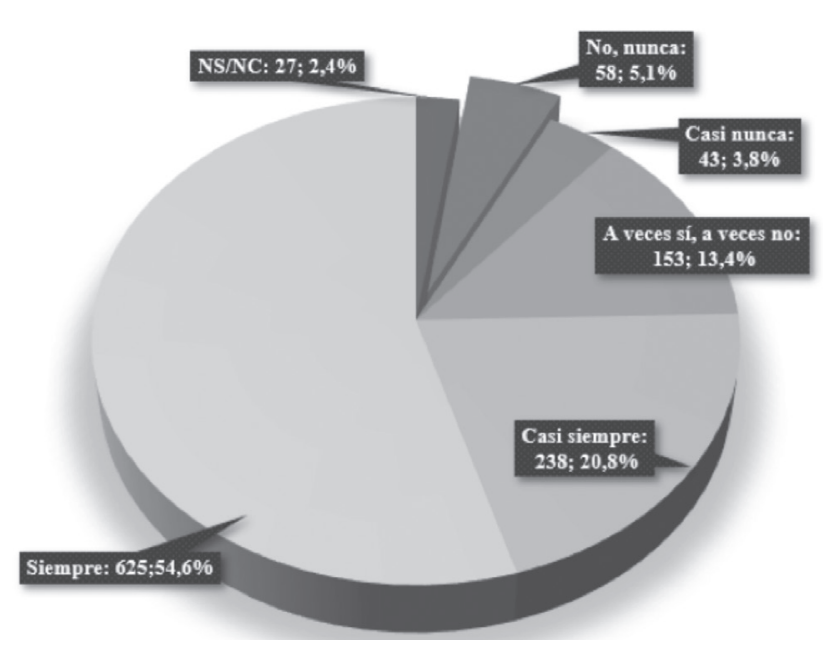

Figura 4. Frecuencias y porcentajes del item "Si tus padres supieran qué haces en las Redes Sociales ite dejarían conectarte a ellas?"

Otro elemento sobre el que se ha profundizado es el que atañe a identificar la opinión que los adolescentes tienen de las redes sociales, y la imagen que ellos piensan que estas herramientas tecnológicas tienen para sus padres y para sus profesores. Como se aprecia en la tabla 1, son los propios estudiantes quienes manifiestan tener una visión de carácter más positivo hacia las redes sociales, mientras que consideran que tanto el profesorado como sus padres no poseen una opinión clara acerca de las mismas (alcanzando porcentajes de un $47,6 \%$ y un $54,8 \%$, respectivamente). Asimismo, los estudiantes piensan, respecto al colectivo docente, que casi un $20 \%$ tiene una visión negativa sobre las redes sociales, porcentaje muy similar $(15,7 \%)$ al alcanzado en el caso de las familias.
Tabla 1.

Frecuencias y porcentajes relativos a la opinión/imagen que para los adolescentes, para sus padres y para el profesorado tienen las redes sociales

\begin{tabular}{lrrrrrr}
\hline & \multicolumn{2}{c}{ Para mi } & \multicolumn{2}{c}{ Para tus padres } & \multicolumn{2}{c}{$\begin{array}{c}\text { Para el } \\
\text { profesorado }\end{array}$} \\
\hline & N & \% & N & $\%$ & $\mathbf{n}$ & $\%$ \\
NS/NC & 23 & 2 & 25 & 2,3 & 43 & 3,7 \\
Muy negativa & 19 & 1,7 & 38 & 3,3 & 83 & 7,3 \\
Negativa & 25 & 2,2 & 180 & 15,7 & 226 & 19,8 \\
Sin opinión & 151 & 13,2 & 545 & 47,6 & 627 & 54,8 \\
Positiva & 593 & 51,8 & 319 & 27,9 & 145 & 12,7 \\
Muy positiva & 333 & 29,1 & 37 & 3,2 & 20 & 1,7 \\
Total & 1144 & $100 \%$ & 1144 & $100 \%$ & 1144 & $100 \%$ \\
\hline
\end{tabular}

\subsection{Análisis estadísticos bivariados de las variables analizadas}

Con el fin de identificar diferencias significativas entre las variables recopiladas en la figura 5, se han llevado a cabo diversos análisis estadísticos bivariados. Es preciso indicar que, en algunos casos, como el relativo a la variable dependiente "rendimiento académico", se han dicotomizado las categorías a "suspenso" y "aprobado", aspecto que ha resultado necesario a fin de disminuir los outliers y evitar frecuencias esperadas inferiores a 5 en el proceso de elaboración de las tablas de contingencia para su posterior contraste con Chi-Cuadrado. También se ha procedido a una recodificación a tres categorías (nunca o casi nunca; a veces sí y a veces no; siempre o casi siempre) de la variable dependiente "Si tus padres supieran qué haces en las Redes Sociales ¿te dejarían conectarte a ellas?".

\begin{tabular}{|l|}
\hline Variables Independientes \\
\hline - Género \\
\hline - Tipo de centro \\
\hline - Nivel de estudios de los padres \\
\hline - $\begin{array}{l}\text { Rendimiento académico } \\
\text { (calificaciones) }\end{array}$ \\
\hline
\end{tabular}

Figura 5. Variables de contraste

\section{Variables Dependientes}

- ¿Quién te invitó a formar parte de tu primera Red Social?

- ¿Quién decide el tiempo que puedes conectarte a las redes sociales?

- ¿Tus padres deciden si te dejan conectarte a las Redes Sociales como premio o castigo por tu comportamiento, tus calificaciones, etc.?

- Si tus padres supieran qué haces en las Redes Sociales ¿te dejarian conectarte a ellas?

- ¿Cuál es la imagen que los adolescentes tienen de las redes sociales y qué percepción tienen acerca de la opinión que tienen sus padres y profesores sobre estas herramientas? 
A continuación, en la tabla 2, se muestra una síntesis del contraste de variables realizado a través de la prueba Chi-Cuadrado.

Tabla 2.

Resumen del grado de significación asintótica (bilateral) tras la aplicación de la prueba Chi-cuadrado

\begin{tabular}{lccccc}
\hline & Variable $1^{*}$ & Variable $2^{*}$ & Variable $3^{*}$ & Variable $4^{*}$ & Variable $5^{*}$ \\
\hline Género & NS & $\mathrm{p}<0,001^{* *}$ & $\mathrm{p}<0,001^{* *}$ & $\mathrm{p}<0,001^{* *}$ & $\mathrm{p}<0,005^{* *}$ \\
Tipo de centro & NS & NS & $\mathrm{p}<0,001^{* *}$ & $\mathrm{NS}$ & NS \\
Nivel de estudios de los padres & NS & NS & NS & NS & NS \\
Rendimiento académico (calificaciones) & NS & NS & $\mathrm{p}<0,005^{* *}$ & $\mathrm{p}<0,005^{* *}$ & NS \\
\hline
\end{tabular}

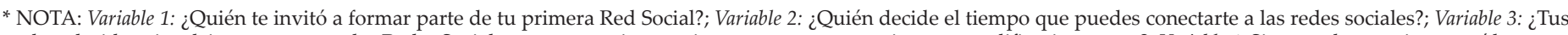

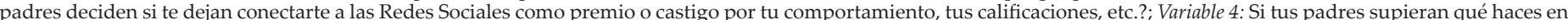

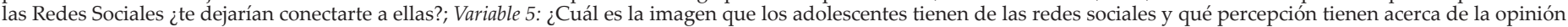
que tienen sus padres y profesores sobre estas herramientas?.

** Resultados significativos a nivel asintótico (bilateral).

NS= No significativo.

Tomando como base los resultados obtenidos en la tabla precedente, se puede decir que en lo que atañe al género, son los chicos frente a las chicas quienes deciden por sí mismos el tiempo de conexión a las redes sociales. Por el contrario, son las chicas quienes manifiestan que el tiempo de conexión depende en mayor medida de la negociación con sus padres, siendo estos más permisivos con las chicas que con los chicos a la hora de limitar el tiempo de conexión.

Estos resultados guardan además un cierto paralelismo respecto a la variable " ¿Tus padres deciden si te dejan conectarte a las Redes Sociales como premio o castigo por tu comportamiento, tus calificaciones, etc.?, donde también se encuentran diferencias significativas por género. Más específicamente, se puede decir que los padres son más restrictivos con los hijos (frente a las hijas), a la hora de permitir conectarse a las redes sociales. Un panorama similar lo encontramos en la variable relativa a "Si tus padres supieran qué haces en las Redes Sociales ¿te dejarían conectarte a ellas?", ya que son las chicas quienes manifiestan en mayor medida (frente a los varones) que sus progenitores casi siempre o siempre les permitirían conectarse.

En lo que atañe al tipo de centro, no se han hallado diferencias estadísticamente significativas, a excepción de la variable “ ¿Tus padres deciden si te dejan conectarte a las Redes Sociales como premio o castigo por tu comportamiento, tus calificaciones, etc.?" (p-valor=.004). En concreto, son los padres de los alumnos de centros públicos, en comparación con los padres de estudiantes de centros concertados y privados, quienes casi siempre o siempre tomarían la decisión dependiendo del comportamiento de los hijos. Otro aspecto analizado fue si el nivel de estudios de los padres podría ser un elemento que tuviese relación con las variables dependientes analizadas. Sin embargo, no se han hallado diferencias estadísticamente significativas.

En cuanto al rendimiento académico (calificaciones), se han encontrado diferencias entre los alumnos que han suspendido alguna materia, frente a los que han aprobado todo, en relación a la variable " ¿Tus padres deciden si te dejan conectarte a las Redes Sociales como premio o castigo por tu comportamiento, tus calificaciones, etc.? (p-valor<.005)". En concreto, son los padres de los estudiantes que suspenden los que casi siempre o siempre toman la decisión de no permitir a sus hijos conectarse a las redes sociales, frente al alumnado que ha aprobado. Asimismo, tras la aplicación de la prueba Chi-cuadrado, se ha encontrado un p-valor inferior a .005 entre el rendimiento académico y la variable "Si tus padres supieran qué haces en las Redes Sociales ¿te dejarían conectarte a ellas?", siendo los padres de los estudiantes que han aprobado los que casi siempre o siempre les dejarían conectarse a las redes sociales, mientras que los progenitores de los alumnos que han suspendido se lo permiten en menor medida en comparación con los aprobados.

Finalmente, con el fin de conocer el tipo de opinión (muy negativa, negativa, sin opinión, positiva, muy positiva) de los propios adolescentes y de lo que ellos pensaban respecto a la imagen que tendrían sus padres y profesores sobre las redes sociales, se elaboró una prueba Chi-Cuadrado, la cual arrojó un resultado significativo (p-valor=.000), por lo que se procedió a la realización de un análisis de correspondencias simple para averiguar cómo se estructura la relación entre las variables y de este modo explorar proximidades entre las mismas.

La tabla 3 recoge un resumen de este análisis de correspondencias simple. Como se puede observar, se han establecido dos dimensiones, siendo la primera de ellas, con un $97,2 \%$, la que explica una mayor proporción de inercia. Igualmente, el total de inercia obtenido es de 0.377 . Siguiendo a Rodríguez Jaume y Mora (2001, p.45) "cuanto mayor sea la inercia total más dependientes serán las variables y más sentido tendrá identificar qué o cuáles categorías (una vez identificados los factores) son las que participan con mayor protagonismo en esa dependencia".

Tabla 3.

Resumen del análisis de correspondencia simple

\begin{tabular}{|c|c|c|c|c|c|c|c|c|}
\hline \multirow[b]{2}{*}{ Dimensión } & \multirow[b]{2}{*}{ Valor propio } & \multirow[b]{2}{*}{ Inercia } & \multirow[b]{2}{*}{ Chi-cuadrado } & \multirow[b]{2}{*}{ Sig. } & \multicolumn{2}{|c|}{ Proporción de inercia } & \multicolumn{2}{|c|}{ Confianza para el Valor propio } \\
\hline & & & & & Explicada & Acumulada & Desviación típica & Correlación 2 \\
\hline 1 & 0,605 & 0,366 & & & 0,972 & 0,972 & 0,013 & 0,046 \\
\hline 2 & 0,102 & 0,010 & & & 0,028 & 1,000 & 0,015 & \\
\hline Total & & 0,377 & 1258,900 & $0,000^{\mathrm{a}}$ & 1,000 & 1,000 & & \\
\hline
\end{tabular}

a: 8 grados de libertad 
La figura 6 muestra un mapa perceptual en el que se interrelacionan las opiniones respecto a las redes sociales de alumnado, padres y profesorado. Como se puede apreciar en dicha figura los profesores (en opinión del alumnado) tienen una imagen fundamentalmente negativa de las redes sociales y también manifiestan no tener una opinión clara sobre las mismas, es decir, se sitúan tal y como se observa en la figura 6 más cerca del atributo "sin opinión". En una posición similar se encuentran los padres que tampoco tienen muy claro lo positivo o negativo que supone el uso de estas herramientas comunicativas. Sin embargo, como se puede apreciar en la citada figura, es el alumnado quien posee una visión más positiva de las redes sociales. Asimismo, es preciso señalar que la opinión "muy negativa" es escasa en los tres colectivos, ya que como se observa en el mapa perceptual se aleja del eje de coordenadas.

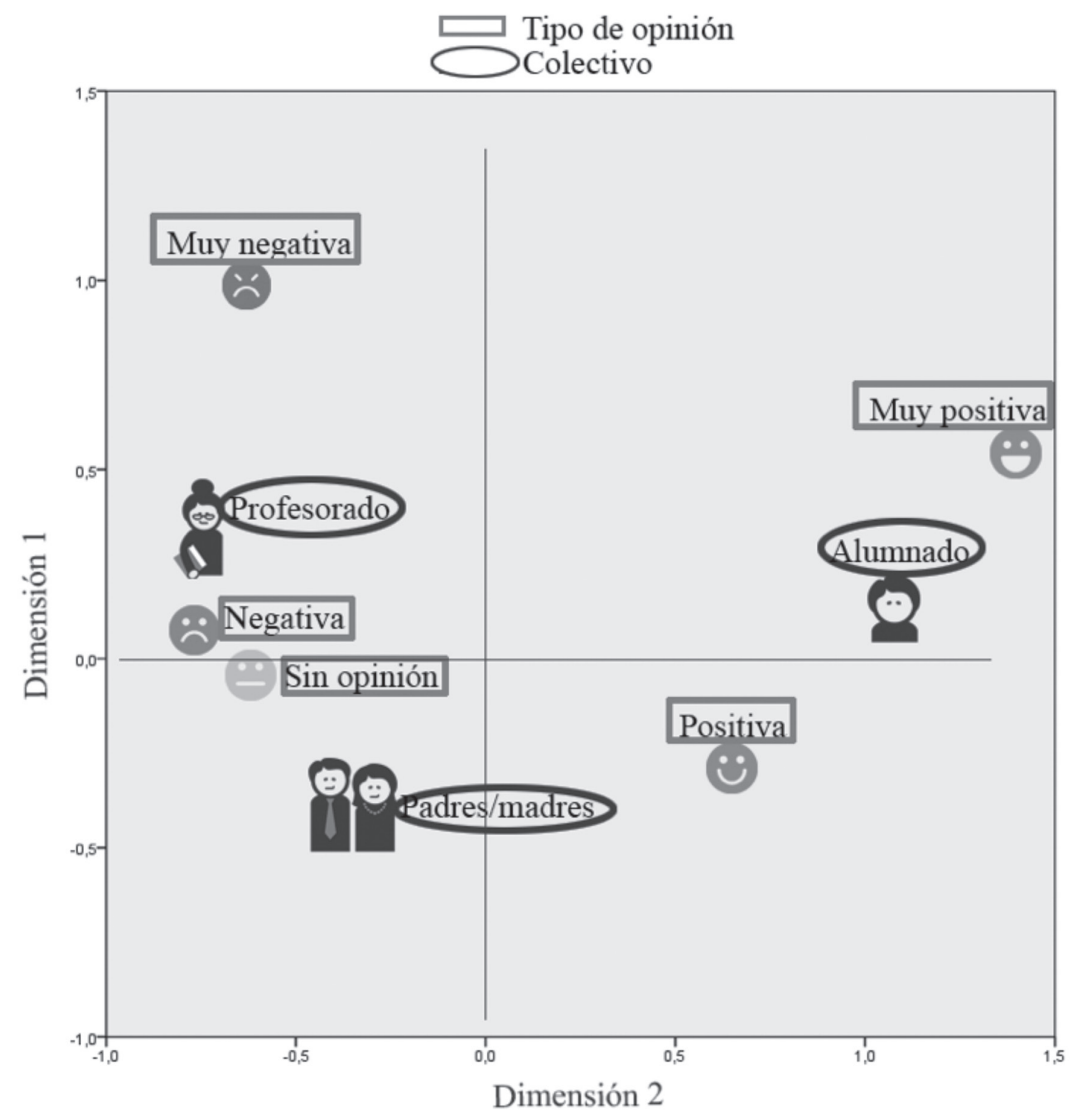

Figura 6. Mapa perceptual con puntos de columna y de fila del análisis de correspondencias simple

\section{Discusión y conclusiones}

La educación digital representa un reto dinámico para las familias tanto por su complejidad como por las múltiples implicaciones que suscita. Comprender el fenómeno de las redes sociales resulta imprescindible para hacerlas fructificar desde una perspectiva formativa e incluso, desde una posición más drástica, cuestionar su uso como señalan Christakis et al. (2010) o Naval et al. (2016). Las variables analizadas en este estudio permiten profundizar en algunos de los aspectos controvertidos respecto al binomio familias y redes sociales, contribuyendo a explorar el papel de los padres con relación al uso que los adolescentes hacen de estas herramientas.

La invitación para usar las redes incluye a padres, amigos y profesores. Destaca mayoritariamente el mundo de los iguales $(72,5 \%)$, pues los adolescentes acceden a la red y se mantienen en línea a través de los pares. En cuanto a la decisión del tiempo, en consonancia con otros estudios (Colás et al., 2013; Rial et al., 2014), queda patente la gran autonomía de la que disponen ya que un $55,2 \%$ lo deciden ellos mismos. Tal como argumentan Chan et al. (2015) y Taddicken (2014), a los padres les preocuparía más la duración que el contenido, pero tampo- co ponen medios efectivos para lograr cambios en el tiempo de conectividad.

En cuanto a las redes sociales como instrumento de control o sanción de los padres, esto es, como premio o castigo, los resultados revelan que en los hogares sí hay cierta negociación, pues los alumnos que suspenden tienen menos permiso para conectarse. De hecho, Liu, Kirschner y Karpinski (2017) advierten de la relación negativa entre estudiantes de bajo rendimiento y uso de redes sociales. Sin embargo, conviene hacer notar con Kirschner (2015) y Muñoz-Carril, Dans-Álvarez-de-Sotomayor y González-Sanmamed (2019) que el uso de las redes sociales en el entorno académico es todavía escaso, lo que resta posibilidades educativas a estas herramientas. En ese mismo sentido, este trabajo confirma la necesidad de un empeño activo por parte de las familias educadoras para llenar el vacío en la resolución de problemas con redes sociales.

Sorprende el dato de que son las chicas las que disponen de más facilidades de conexión frente a los chicos. Estas diferencias a nivel de género también han sido constatadas en otras investigaciones como las desarrolladas por Alonso-Ruido, Rodríguez-Castro, Lameiras-Fernández, y Carrera-Fernández (2015). 
La última variable incluida se refiere a la visión global de los adolescentes sobre las redes sociales e incorpora su juicio sobre la imagen y percepción que tendrían los adultos: padres y profesores. Los adolescentes manifiestan rotundamente $(72,5 \%)$ tener una imagen positiva sobre las redes sociales, que decrece respecto a la que tendrían sus padres y, menos aún, la de los profesores, a quienes se les atribuye una imagen más negativa sobre estas herramientas. Este último dato refuerza la idea de que redes sociales y escuela son dos conceptos distantes en la mente de los jóvenes, pero no así en el horizonte doméstico. Así pues, podría afirmarse que los datos analizados contribuyen a vislumbrar un papel nuclear de la familia en la educación digital, y no solo por las responsabilidades que han de asumir en cualquier asunto relativo a la educación de sus hijos, sino que son los mismos adolescentes los que otorgan mayor protagonismo a sus padres y madres frente a sus profesores en las relaciones con las redes sociales.

Conocer las condiciones de uso y control sobre las redes sociales en el ámbito familiar permite aclarar las circunstancias que rodean a los jóvenes en su vida digital. Así, una auténtica alfabetización digital pasaría por hacer conscientes a padres e hijos como participantes activos en el uso educativo de las redes sociales. En este sentido, resulta también importante el desarrollo de estrategias de co-responsabilidad entre la escuela y la familia, a fin de desarrollar sinergias que propicien una adecuada "cibersocialización" de los adolescentes (Pérez-Bonet, 2010). En esta línea, las Escuelas de Padres pueden resultar un mecanismo válido para propiciar no solo una mayor participación y comunicación bidireccional entre las familias y los centros educativos, sino también para implementar acciones formativas que permitan a los padres mejorar sus conocimientos tecnológicos y desarrollar con sus hijos estrategias de mediación parental eficaces que contribuyan a un uso adecuado de la Red. En definitiva, tal y como recomiendan Mayorgas (2009) y Rial et al. (2014), es preciso que las familias tomen conciencia de la utilización que hacen sus hijos de las redes sociales, desarrollando medidas que supongan un control responsable y una orientación más eficaz de las posibilidades de uso de internet.

\section{Referencias}

Almu, A. y Buhari, B. (2014). Effect of Mobile Social Networks on Secondary School Students. International Journal of Computer Science and Information Technologies, 5(5), 6333-6335. Recuperado de http://ijcsit.com/docs/Volume\%205/vol5issue05/ijcsit2014050574.pdf.

Alonso-Ruido, P., Rodríguez-Castro, Y., Lameiras-Fernández, M., y Carrera-Fernández, M. (2015). Hábitos de uso en las Redes Sociales de los y las adolescentes: análisis de género. Revista de Estudios e Investigación en Psicología y Educación, 13, 54-57. https://doi.org/10.17979/reipe.2015.0.13.317.

Ballesta, F.J., Lozano, J., Cerezo, M.C. y Soriano, E. (2015). Internet, redes sociales y adolescencia: un estudio en centros de educación secundaria de la región de Murcia. Revista Fuentes, 16, 109-130. Recuperado de https://revistascientificas.us.es/ index.php/fuentes/article/view/2365.

Bicen, H. y Uzunboylu, H. (2013). The Use of Social Networking Sites in Education: A Case Study of Facebook. Journal of Universal Computer Science, 19(5), 658-671. https://www.doi. org/10.3217/jucs-019-05-0658.

Bringué, X. y Sádaba, C. (2011). Menores y redes sociales. Madrid: Foro Generaciones Interactivas.

Bronfenbrenner, U. (1994). Ecological models of human development. En International Encyclopedia of Education, 3 (2 $2^{-}$ed.). Oxford, UK: Elsevier.
Busquet, J., Medina, A. y Ballano, S. (2013). El uso de las TRIC y el choque cultural en la escuela. Encuentros y desencuentros entre maestros y alumnos. Revista Mediterránea de Comunicación, 4(2), 115-135. https://doi.org/10.14198/MEDCOM2013.4.2.06.

Carr, N. (2011). Superficiales: ¿Qué está haciendo internet con nuestras mentes?. Madrid: Taurus.

Chan, N. Walker, C. y Gleaves, A. (2015). An exploration of students' lived experiences of using smartphones in diverse learning contexts using a hermeneutic phenomenological approach. Computers y Education, 82, 96-106. https://doi.org/10.1016/j.compedu.2014.110,001.

Christakis, N. A., Fowler, J. H., Diéguez, A., Vidal, L., y Schmid, E. (2010). Conectados: el sorprendente poder de las redes sociales y cómo nos afectan. Madrid: Taurus.

Colás, P., González, T., y De Pablos, J. (2013). Young People and Social Networks: Motivations and Preferred Uses. Comunicar, 40, 15-23. https://www.doi.org/10.3916/C40-2013-02-01.

Commons Sense Media (2010). The common sense census: media use by tweens and teens. Recuperado de https://www.commonsensemedia.org/sites/default/files/uploads/research/census_researchreport.pdf.

Commons Sense Media (2018). 2017-2018 annual report. Recuperado de https://www.commonsensemedia.org/sites/default/ files/uploads/pdfs/annual-report-web-spreads.pdf.

Davis, G. (2018). The Age of Content. McAffe. Recuperado de https://securingtomorrow.mcafee.com/consumer/consumer-threat-notices/age-of-consent-survey-insights/

Durkee, T., Kaess, M., Carli, V., Parzer, P., Wasserman, C., Floderus, B. y Wasserman, D. (2012). Prevalence of pathological Internet use among adolescents in Europe: Demographic and social factors. Addiction, 107(12), 2210-2222. https://www.doi. $\operatorname{org} / 10.1111 / \mathrm{j} .1360-0443.2012 .03946 . x$.

García-Ruiz, R.; Tirado, R. y Hernado, A. (2018). Redes sociales y estudiantes: motivos de uso y gratificaciones. Evidencias para el aprendizaje, Aula Abierta, 47(3), 291-298. https://doi. org/10.17811/rifie.47.3.2018.291-298.

Garrido, M., Busquet, J. y Munté, R. A. (2016). De las TIC a las TRIC. Estudio sobre el uso de las TIC y la brecha digital entre adultos y adolescentes en España. Anàlisi. Quaderns de Comunicació i Cultura, 54, 44-57. http://dx.doi.org/10.7238/a.v0i54.2953.

González, M., Muñoz, P. y Dans, I. (2017). Factors which motivate the use of social networks by students, Psicothema 29(2), 204-210, https://doi.org/10.7334/psicothema2016.127.

Hamid, S., Waycott, J., Kurnia, S. y Chang, S. (2015). Understanding students' perceptions of the benefits of online social networking use for teaching and learning. The Internet and Higher Education, 26, 1-9. http://www.doi.org/10.1016/j.iheduc.2015.02.004

INTECO. Instituto nacional de tecnologías de la comunicación y Orange (2011). Estudio sobre hábitos seguros en el uso de smartphones por los niños y adolescentes españoles. Recuperado de http://www.inteco.es/guias_estudios/Estudios/Estudio_moviles_3C2011.

Junco, R. (2015). Student class standing, Facebook use and academic performance. Journal of Applied Developmental Psychology, 36, 18-29. https://doi.org/10.1016/j.appdev.2014.110,001.

Jung-Lee, S. (2009). Online Communication and Adolescent Social Ties: Who Benefits More from Internet use? Journal of Computer-Mediated Communication, 14, 509-531. Internet and Higher Education, 26, 1-9. https:/www.doi.org/10.1016/j.iheduc.2015.02.004.

Kirschner, P. y Karpinski, A. (2010). Facebook and academic performance. Computers in Human Behavior, 26, 1237-1245. https://doi.org/10.1016/j.chb.2010.03.024. 
Kirschner, P. (2015). Facebook as learning platform: argumentation superhighway or dead-end street? Computers in Human Behavior, 53, 621-625. https://doi.org/10.1016/j. chb.2015.03.011.

Kuppuswamy, S., y Narayan, P. S. (2010). The impact of social networking websites on the education of youth. International Journal of Virtual Communities and Social Networking, 2(1), 6779. https://www.doi.org/10.4018/jvcsn.2010010105.

Liu, D., Kirschner, P.A., y Karpinski, A.C. (2017). A meta-analysis of the relationship of academic performance and Social Network Site use among adolescents and young adults. Computers in Human Behavior, 77, 148-157. https://www.doi. org/10.1016/j.chb.2017.08.039.

Livingstone, S. y Haddon, L. (2011). EU Kids Online III. A new project. London: LSE, EU Kids Online. Recuperado de http:// www2.lse.ac.uk/media@lse/research/EUKidsOnline/Home. as.

Livingstone, S., Blum-Ross, A. y Zhang, D. (2018) What do parents think, and do, about their children's online privacy? Parenting for a Digital Future: Survey Report 3, Department of Media and Communications. London, UK: The London School of Economics and Political Science. Recuperado de http://eprints. lse.ac.uk/87954/1/Livingstone_Parenting\%20Digital\%20Survey\%20Report\%203_Published.pdf.

López Moratalla, N., Bernar, C., Sueiro, E. y Valderas, J.M. (2011). Los secretos de tu cerebro. Cerebro adolescente: de aislarse a intimar. Navarra: Universidad de Navarra. Recuperado de http://www.unav.es/servicio/comunicacioncientifica/cerebroadolescente.

Mayorgas, M. J. (2009). Programas de prevención de la adicción a las nuevas tecnologías en jóvenes y adolescentes. En E. Echeburúa, F.J. Labrador y E. Becoña (Eds.), Adicción a las nuevas tecnologías en adolescentes y jóvenes (pp. 221-249). Madrid: Pirámide.

McMillan, J. y Schumacher, S. (2005). Investigación educativa. Madrid: Pearson Addison Wesley.

Moeller, S., Powers, E., y Roberts, J. (2012). «The World Unplugged» and «24 Hours without Media»: Media Literacy to Develop Self-Awareness Regarding Media. Comunicar, 39, 4552. https://doi.org/10.3916/C39-2012-02-04.

Moratalla, A. D. (2008). Familia, escuela y sociedad: responsabilidades compartidas en la educación. Madrid: Ediciones Cinca.

Mullen, C. y Hamilton, N. F. (2016). Adolescents' response to parental Facebook friend requests: The comparative influence of privacy management, parent-child relational quality, attitude and peer influence. Computers in Human Behavior, 60, 165-172. http://dx.doi.org/10.1016/j.chb.2016.02.026.

Muñoz-Carril, P.C., Dans-Álvarez-de-Sotomayor, I. y González-Sanmamed, M. (2019). Social Networks and Their Uses in The Field Of Secondary Education. En N. Dey, S. Borah, R. Babo y A. Ashour (Eds.), Social Network Analytics. Computational Research Methods and Techniques (pp. 203-226). London: Academic Press. Elsevier Inc.

Naval, C., Serrano-Puche, J., Sádaba, C. y Arbués, E. (2016). Sobre la necesidad de desconectar: algunos datos y propuestas. Education in the Knowledge Society, 17(2), 73-90. http://dx.doi. org/10.14201/eks20161727390.

Norton (2018). Cyber Security Insights 2017. Recuperado de https://us.norton.com/cyber-security-insights-2017.

Pew Research Center (2018). How Teens and Parents Navigate Screen Time and Device Distractions. Recuperado de http://www. pewinternet.org/2018/08/22/how-teens-and-parents-navigate-screen-time-and-device-distractions/
Pérez-Bonet, G. (2010). Cibersocialización y adolescencia: un nuevo binomio para la reflexión en educación social. Revista de Educación Social, 11. Recuperado de http://www.eduso.net/ res/?b=14\&c=129\&n=367.

Rial, A., Gómez, P., Braña, T. y Varela, J. (2014). Actitudes, percepciones y uso de las redes sociales entre los adolescentes de la comunidad gallega (España). Anales de Psicología, 30(2), 642-655. https://doi.org/10.6018/analesps.30.2.159111.

Ródenas-Rigla, F. J., Fombuena, J. y Pérez-Cosín, J. V. (2017) (Coords.). Bienestar social: intervención familiar. Valencia: Tirant lo Blanch.

Rodríguez Jaume, M. J. J. y Mora Catalá, R. (2001). Estadística informática: casos y ejemplos con el SPSS. Alicante: Servicio de Publicaciones Universidad de Alicante.

Ruiz-Corbella, M. y De-Juanas, A. (2013). Redes sociales, identidad y adolescencia: nuevos retos educativos para la familia. Estudios sobre Educación, 25, 95-113. Recuperado de https://www.unav.edu/publicaciones/revistas/index.php/ estudios-sobre-educacion/article/view/1883.

Salcines-Talledo, I., Ramírez, A. y González-Fernández, N. (2018). Smartphones y tablets en familia. Diseño de un instrumento diagnóstico. Aula Abierta, 47(3), 265-272. https://doi. org/10.17811/rifie.47.3.2018.265-272

Salmerón, M. A. (2015). Influencia de las TIC en la salud del adolescente. Adolescere. Revista de Formación Continuada de La Sociedad Española de Medicina de la Adolescencia, 3(2), 18-25. Recuperado de https:/www.adolescenciasema.org/wp-content/ uploads/2015/06/adolescere-2015-vol3-n2_18-25_Influencia_ de_las_TIC.pdf.

Shin, W. y Kang, H. (2016). Adolescents' privacy concerns and information disclosure online: The role of parents and the Internet. Computers in Human Behavior. 54, 114-123. http://doi. org/10.1016/j.chb.2015.07.062.

Taddicken, M. (2014). The 'privacy paradox'in the social web: The impact of privacy concerns, individual characteristics, and the perceived social relevance on different forms of self disclosure. Journal of Computer Mediated Communication, 19(2), 248-273. doi: 10.1111/jcc4.12052

Thuseethan, S. y Kuhanesan, S. (2014). Positive and Negative Impacts of Facebook on University Students: SriLankan Perspective. The IUP Journal Of Information Technology, 10(3), 718. Recuperado de https://goo.gl/x0XKhc.

Valkenburg, P. M., y Peter, J. (2011). Online communication among adolescents: An integrated model of its attraction, opportunities, and risks. Journal of adolescent health, 48(2), 121127. https://www.doi.org/10.1016/j.jadohealth.2010.08.020.

Vázquez, T. D., Hurtado, M. J. R., y Baños, R. V. (2018). La adolescencia ante la violencia de género 2.0: Concepciones, conductas y experiencias. Educación XX1, 21(1), 109-133. https:// doi.org/10.5944/educxx1.20180.

Vilà, R. y Bisquerra, R. (2004). El análisis cuantitativo de los datos. En R. Bisquerra (Coord.), Metodología de la investigación educativa (pp. 259-271). Madrid: Editorial la Muralla.

\section{Agradecimientos}

Artículo elaborado en el marco de la investigación titulada: “Cómo aprenden los mejores docentes universitarios en la era digital: impacto de las ecologías de aprendizaje en la calidad de la docencia" (ECO4LEARN-HE), con Referencia EDU201567907-R, financiado parcialmente por el MINECO. 
